\title{
Numerical Simulations of Magnetoacoustic-Gravity Waves in the Solar Atmosphere
}

\author{
K. Murawski • A.K. Srivastava · J.A. McLaughlin • \\ R. Oliver
}

Received: 25 May 2012 / Accepted: 24 November 2012 / Published online: 21 December 2012

(C) The Author(s) 2012. This article is published with open access at Springerlink.com

\begin{abstract}
We investigate the excitation of magnetoacoustic-gravity waves generated from localized pulses in the gas pressure as well as in the vertical component of velocity. These pulses are initially launched at the top of the solar photosphere, which is permeated by a weak magnetic field. We investigate three different configurations of the background magnetic field lines: horizontal, vertical, and oblique to the gravitational force. We numerically model magnetoacoustic-gravity waves by implementing a realistic (VAL-C) model of the solar temperature. We solve the two-dimensional ideal magnetohydrodynamic equations numerically with the use of the FLASH code to simulate the dynamics of the lower solar atmosphere. The initial pulses result in shocks at higher altitudes. Our numerical simulations reveal that a small-amplitude initial pulse can produce magnetoacoustic-gravity waves, which are later reflected from the transition region due to the large-temperature gradient. The cavities in the lower solar atmosphere are found to have the best conditions to act as a resonator for various oscillations, including their trapping and leakage into the higher atmosphere. Our
\end{abstract}

Electronic supplementary material The online version of this article

(doi:10.1007/s11207-012-0202-7) contains supplementary material, which is available to authorized users.

K. Murawski $(\varangle)$

Faculty of Mathematics, Physics, and Informatics, UMCS, ul. Radziszewskiego 10, 20-031 Lublin, Poland

e-mail: kmur@kft.umcs.lublin.pl

A.K. Srivastava

Aryabhatta Research Institute of Observational Sciences (ARIES), Nainital, India e-mail: aks@aries.res.in

J.A. McLaughlin

School of Computing, Engineering and Information Sciences, Northumbria University,

Newcastle Upon Tyne NE1 8ST, UK

e-mail: james.a.mclaughlin@northumbria.ac.uk

R. Oliver

Departament de Física, Universitat de les Illes Balears, 07122 Palma de Mallorca, Spain

e-mail: ramon.oliver@uib.es 
numerical simulations successfully model the excitation of such wave modes, their reflection and trapping, as well as the associated plasma dynamics.

Keywords Waves $\cdot$ Magnetic fields $\cdot$ Corona $\cdot$ Granulation

\section{Introduction}

The complicated magnetic-field configuration of the Sun plays a key role in various types of dynamical plasma processes in its atmosphere, including all significant plasma dynamics of the lower solar atmosphere. The resulting magnetic structures channel energy from the photosphere into the upper atmosphere in the form of magnetohydrodynamic (MHD) waves. These waves experience mode conversion, resonances, trapping, and reflection, which result in the complicated dynamical processes in the lower solar atmosphere, the details of which depend on the plasma properties as well as on the strength of the magnetic field. The complex magnetic-field and plasma structuring in the lower solar atmosphere support the excitation of various kinds of MHD waves. Their propagation, reflection, and trapping have been extensively studied theoretically and observationally (e.g. McAteer et al., 2003; Hasan et al., 2005; Srivastava et al., 2008; Fedun, Erdélyi, and Shelyag, 2009; Srivastava, 2010; Murawski, Srivastava, and Zaqarashvili, 2011; Gruszecki et al., 2011; and references therein). The evolving magnetic fields of the lower solar atmosphere also lead to transient processes across a wide range of spatial-temporal scales in the form of eruption and associated phenomena. For example, various types of solar jets are formed at short spatialtemporal scales, which play a significant role in mass and energy transport and also couple the various layers of the solar atmosphere (Shibata et al., 2007; Katsukawa et al., 2007; Srivastava and Murawski, 2011; and references therein). In addition, the magnetic activity and injections of helicity into the lower solar atmosphere result in large-scale eruptive phenomena, including solar flares and coronal mass ejections (CMEs) in the outer part of the magnetized solar atmosphere (Srivastava et al., 2010; Shibata and Magara, 2011; Zhang, Cheng, and Ding, 2012; and references therein). All this makes the coupling of the complex magnetic field in various layers of the Sun, caused by waves and transients, one of the most significant areas of contemporary solar research.

In the quiet-Sun magnetic networks, cavities are important locations where the magnetic fields are sufficiently inclined due to their well-evolved horizontal components. The cavities are formed over the granular cells in the form of field-free regions through the transport of plasma at their boundaries, and are overlaid by bipolar magnetic canopies. The vertical magnetic fields, however, reside mostly in the core of these magnetic networks (Schrijver and Title, 2003; Centeno et al., 2007). The magnetic cavity-canopy systems are thought to be ideal resonators of the various MHD waves that can be trapped in the cavity, and can also leak upward through the core of these magnetic networks in the form of magnetoacoustic-gravity waves. It is thought that the field-free cavity regions underlying the bipolar canopy can trap the high-frequency acoustic oscillations, and the low-frequency components may leak into the higher atmosphere in the form of magnetoacoustic-gravity waves (Kuridze et al., 2008; Srivastava et al., 2008; Srivastava, 2010). Therefore, these magnetic structures in the lower solar atmosphere may play an important role in wave filtering (McIntosh and Judge, 2001; Krijger et al., 2001; McAteer et al., 2002; Vecchio et al., 2007; Vecchio, Cauzzi, and Reardon, 2009; Srivastava, 2010; and references therein).

In addition to recent high-resolution observations of MHD waves in the lower solar atmosphere, extensive efforts have been made in the area of analytical and numerical modeling 
of these waves: Fedun, Erdélyi, and Shelyag (2009) have investigated the 3D numerical modeling of the coupled slow and fast magnetoacoustic wave propagation in the lower solar atmosphere; and recently Fedun et al. (2011) have reported the first numerical results of the frequency filtering of torsional Alfvén waves in the chromosphere. In addition to general numerical modeling of the waves in the lower solar atmosphere, models have also investigated the acoustic-wave spectrum in the localized magnetic structures of the lower solar atmosphere, e.g. the magnetic cavity-canopy system (Kuridze et al., 2008; Srivastava et al., 2008; Kuridze et al., 2009; and references cited therein).

It is also noteworthy that Bogdan et al. (2003), Fedun, Erdélyi, and Shelyag (2009), and Fedun et al. (2011), discussed in detail the excitation, propagation, and conversion of magnetoacoustic waves in a realistic 3D MHD simulation. However, in these references the waves driven by a periodic driver were discussed, whereas we numerically simulate the excitation of magnetoacoustic-gravity waves generated by pulses in the gas pressure and the vertical velocity component, which mimics an isolated solar granule. We aim to investigate and understand this simpler (but still complex enough) system before we move on to the more realistic, multiple-granule system. Our philosophy is to build up our models incrementally, with a clear focus on the underlying physical processes at each step.

We here investigate the excitation of magnetoacoustic-gravity waves generated from localized pulses in the gas pressure as well as in the vertical velocity component by modeling the effect of an isolated solar granule. These pulses are initially launched at the top of the solar photosphere, which is permeated by a weak magnetic field. We investigate three different configurations of the background magnetic-field lines: vertical, horizontal, and oblique to the gravitational force. We aim to show that small-amplitude perturbations that are associated with such a granule are able to trigger large-amplitude, complicated oscillations in the solar corona, which exhibit periodicities within the detected range of three to five minutes.

The structure of the article is as follows: In Section 2 we describe the numerical model. We report the numerical results in Section 3 and present the discussion and conclusions in Section 4.

\section{Numerical Model}

We consider a gravitationally stratified solar atmosphere that is described by the ideal twodimensional (2D) MHD equations:

$$
\begin{aligned}
\frac{\partial \varrho}{\partial t}+\nabla \cdot(\varrho \mathbf{V}) & =0, \\
\varrho \frac{\partial \mathbf{V}}{\partial t}+\varrho(\mathbf{V} \cdot \nabla) \mathbf{V} & =-\nabla p+\frac{1}{\mu}(\nabla \times \mathbf{B}) \times \mathbf{B}+\varrho \mathbf{g}, \\
\frac{\partial p}{\partial t}+\nabla \cdot(p \mathbf{V}) & =(1-\gamma) p \nabla \cdot \mathbf{V}, \\
\frac{\partial \mathbf{B}}{\partial t} & =\nabla \times(\mathbf{V} \times \mathbf{B}), \quad \nabla \cdot \mathbf{B}=0 .
\end{aligned}
$$

Here $\varrho$ is mass density, $\mathbf{V}$ is the flow velocity, $\mathbf{B}$ is the magnetic field, $p=k_{\mathrm{B}} \varrho T / m$ is the gas pressure, $T$ is the temperature, $\gamma=5 / 3$ is the adiabatic index, $\mathbf{g}=(0,-g, 0)$ is the gravitational acceleration, where $g=274 \mathrm{~m} \mathrm{~s}^{-2}, m$ is the mean particle mass, and $k_{\mathrm{B}}$ is Boltzmann's constant. Throughout this article, we use the Cartesian coordinate system with the vertical axis denoted by $y$ and the horizontal axis $x$. Henceforth, we assume that 
the medium is invariant along the $z$-direction with $\partial / \partial z=0$ and set the $z$-components of plasma velocity and magnetic field equal to zero, i.e. $V_{z}=0$ and $B_{z}=0$. This assumption removes Alfvén waves from the system but still allows magnetoacoustic-gravity waves to propagate freely.

\subsection{Equilibrium Configuration}

We assume that the solar atmosphere is static $\left[\mathbf{V}_{\mathrm{e}}=\mathbf{0}\right]$ at its equilibrium, and that is threaded by a straight magnetic field,

$$
\mathbf{B}=B_{0} \hat{\mathbf{s}}
$$

where $\hat{\mathbf{s}}$ is a unit vector that is either vertical, horizontal, or oblique. We choose $B_{0}$ by requiring that at the reference point $(0,10) \mathrm{Mm}$ the Alfvén speed

$$
c_{\mathrm{A}}(y)=\frac{B_{0}}{\sqrt{\mu \varrho_{\mathrm{e}}(y)}}
$$

and sound speed

$$
c_{\mathrm{s}}(y)=\sqrt{\frac{\gamma p_{\mathrm{e}}(y)}{\varrho_{\mathrm{e}}(y)}}
$$

satisfy the constraint $c_{\mathrm{A}}(y=10 \mathrm{Mm})=10 c_{\mathrm{s}}(y=10 \mathrm{Mm})$. This constraint reproduces typical conditions in the solar corona where, typically, $c_{\mathrm{s}}=100 \mathrm{~km} \mathrm{~s}^{-1}$ and $c_{\mathrm{A}}=1000 \mathrm{~km} \mathrm{~s}^{-1}$. As a result, the solar corona is magnetically dominated with $B_{0} \approx 14.5 \times 10^{-4} \mathrm{~T}$. The plasma $\beta$,

$$
\beta(y)=\frac{\gamma}{2} \frac{c_{\mathrm{s}}^{2}(y)}{c_{\mathrm{A}}^{2}(y)},
$$

in the solar corona attains a value of $\beta(y=10 \mathrm{Mm})=0.012$. It slowly grows with depth within the chromosphere and abruptly reaches a value of $\beta(y=0 \mathrm{Mm}) \approx 4 \times 10^{5}$ below $y=2 \mathrm{Mm}$ at the bottom of the photosphere (Figure 1, bottom). This high value of $\beta$ shows that in these low regions of the solar atmosphere the effect of the magnetic field is negligibly small.

The straight magnetic field of Equation (5) is a simplified model of curved magneticfield lines that are located in the low regions of the solar atmosphere. However, because the plasma $\beta$ grows rapidly below $y=2.5 \mathrm{Mm}$, where it reaches high values (see Figure 1, bottom), we in fact model the very weakly magnetized low layers of the atmosphere, which do not correspond to flux-tubes and sunspots. Therefore, in this first approximation, the curved magnetic-field lines can be replaced by a straight magnetic field, and our models are justified.

In Equations (6) and (7), $\varrho_{\mathrm{e}}(y)$ and $p_{\mathrm{e}}(y)$ denote the equilibrium mass density and gas pressure, respectively. They are specified by the hydrostatic constraint, which in the context of Equation (5) states that the pressure gradient is balanced by the gravity force,

$$
-\nabla p_{\mathrm{e}}+\varrho_{\mathrm{e}} \mathbf{g}=\mathbf{0}
$$

With the ideal gas law and the $y$-component of Equation (9), we arrive at

$$
p_{\mathrm{e}}(y)=p_{0} \exp \left[-\int_{y_{\mathrm{r}}}^{y} \frac{\mathrm{d} y^{\prime}}{\Lambda\left(y^{\prime}\right)}\right], \quad \varrho_{\mathrm{e}}(y)=\frac{p_{\mathrm{e}}(y)}{g \Lambda(y)},
$$




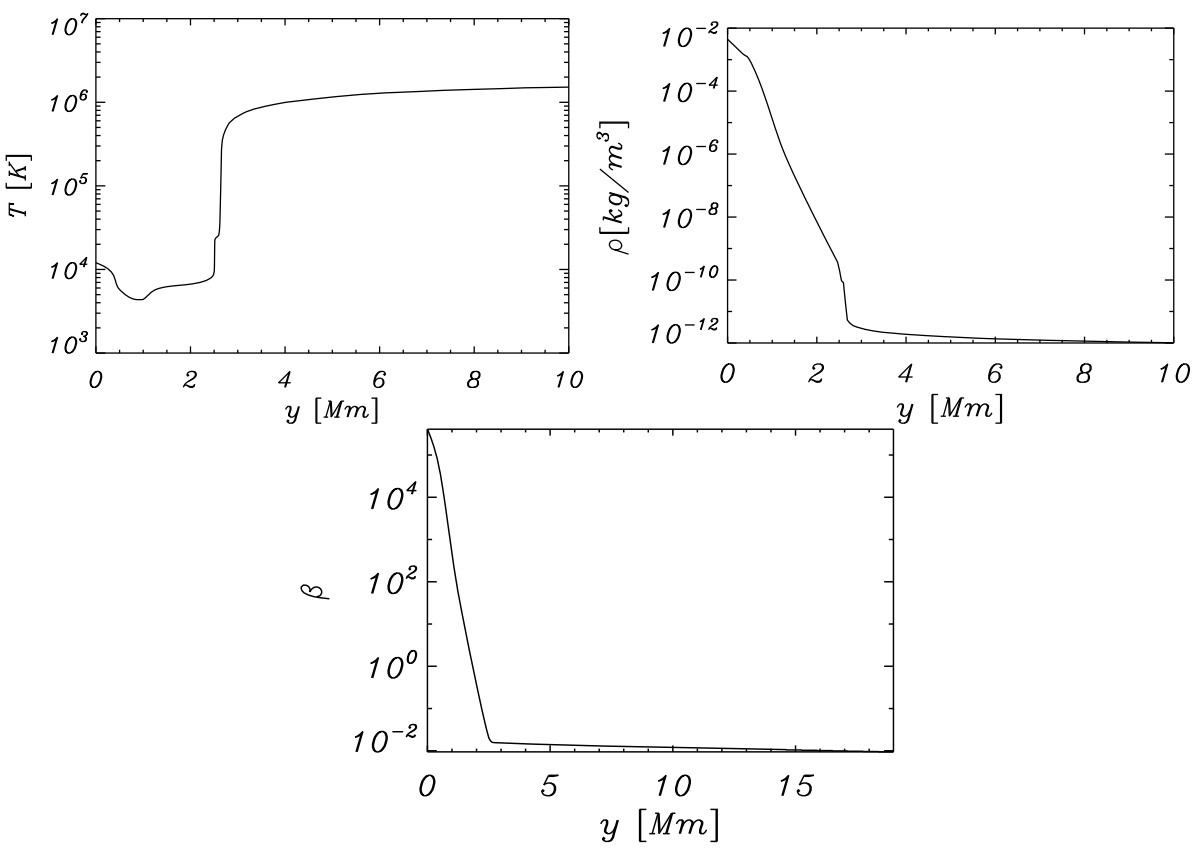

Figure 1 Equilibrium profile of the solar temperature (top-left), the mass density (top-right), and the plasma $\beta$ (bottom).

where

$$
\Lambda(y)=\frac{k_{\mathrm{B}} T_{\mathrm{e}}(y)}{m g}
$$

is the pressure scale-height, and $p_{0}$ denotes the gas pressure at the reference level that we choose in the solar corona at $y_{\mathrm{r}}=10 \mathrm{Mm}$.

We adopt an equilibrium temperature profile $\left[T_{\mathrm{e}}(z)\right]$ for the solar atmosphere that is close to the VAL-C atmospheric model of Vernazza, Avrett, and Loeser (1981): see Figure 1, topleft panel. $T_{\mathrm{e}}$ attains a value of about $5700 \mathrm{~K}$ at the top of the photosphere, which corresponds to $y=0.5 \mathrm{Mm}$. At higher altitudes, $T_{\mathrm{e}}(y)$ falls off until it reaches its minimum of $4350 \mathrm{~K}$ at an altitude of $y \approx 0.95 \mathrm{Mm}$. Higher up, $T_{\mathrm{e}}(y)$ grows gradually with height up to the transition region, which is located at $y \approx 2.7 \mathrm{Mm}$. Here $T_{\mathrm{e}}(y)$ experiences a sudden increase to the coronal value of $1.5 \mathrm{MK}$ at $y=10 \mathrm{Mm}$. Then with Equation (10) we obtain the corresponding gas pressure and mass-density profiles. Both $p_{\mathrm{e}}(y)$ (not shown) and $\varrho_{\mathrm{e}}(y)$ (Figure 1, top-right panel) experience a sudden decrease from photospheric to coronal values at the transition region.

\subsection{Initial Conditions}

At $t=0$ seconds, we initially perturb the equilibrium impulsively by simultaneously using localized Gaussian pulses in the gas pressure and the vertical-velocity component, viz.

$$
p(x, y, t=0)=p_{\mathrm{e}}(y)+A_{\mathrm{p}} f(x, y), \quad V_{y}(x, y, t=0)=A_{\mathrm{v}} f(x, y),
$$




$$
f(x, y)=\exp \left[-\frac{\left(x-x_{0}\right)^{2}}{w_{x}^{2}}-\frac{\left(y-y_{0}\right)^{2}}{w_{y}^{2}}\right]
$$

Here $A_{\mathrm{p}}$ and $A_{\mathrm{v}}$ are the amplitudes of the perturbations, $\left(x_{0}, y_{0}\right)$ is their initial position, and $\left(w_{x}, w_{y}\right)$ denotes their widths along the $x$ - and $y$-directions. We set and hold fixed $x_{0}=$ $0 \mathrm{Mm}, y_{0}=0.5 \mathrm{Mm}, w_{x}=0.5 \mathrm{Mm}, w_{y}=0.5 \mathrm{Mm}, A_{\mathrm{p}}=0.02 p_{\mathrm{e}}\left(y_{0}\right)$, and $A_{\mathrm{v}}=0.2 \mathrm{~km} \mathrm{~s}^{-1}$. These magnitudes of $A_{\mathrm{v}}$ and $A_{\mathrm{p}}$ lie within the range of the detected flow and temperature in solar granulation (e.g. Rieutord et al., 2002; Kostik, Khomenko, and Shchukina, 2009; Baran, 2011). We separately consider three orientations for our unidirectional equilibrium magnetic field lines: (a) horizontal, (b) vertical, (c) oblique to the gravitational force.

\section{Numerical Results}

Equations (1) - (4) are solved numerically using the FLASH code (Lee and Deane, 2009). This code implements a second-order unsplit Godunov solver with various slope limiters and Riemann solvers, as well as adaptive mesh refinement (AMR). The main advantage of using the AMR technique is to refine a numerical grid at steep spatial profiles while keeping a coarse grid at the places where fine spatial resolution is not essential. This technique usually introduces interpolation errors at different-sized numerical cells. These errors can result in a vertical flow that, albeit initially small, can grow with height to an unacceptable magnitude. The remedy we adopted for this inherent phenomenon is to refine the whole region below the transition region. We used the Roe solver and minmod flux limiter, and set the simulation box for the horizontal and vertical equilibrium magnetic field cases in the $x$-direction to be $-5 \leq x \leq 5 \mathrm{Mm}$. To trace plasma structures that extend more horizontally in the case of the oblique magnetic field, we used $-2 \leq x \leq 8 \mathrm{Mm}$. Along the $y$-direction the numerical box was set to $-1 \leq y \leq 19 \mathrm{Mm}$ in all cases. At all boundaries, we fixed all plasma quantities to their equilibrium values, which led only to weak numerical reflections of the incident wave signals. Additionally, we increased the physical domain size and dampened incident waves by adopting a coarse numerical grid at the top boundary to avoid the influence of reflections during the simulation time range. As a result, these reflections do not exert any noticeable effect on the dynamics of the system. In all our studies, we used an AMR grid with a minimum/maximum level of refinement set to 3/7. The initial system of blocks is shown in Figure 2. For the initial vertical and horizontal magnetic fields, at $t=0$ seconds the whole simulation region is covered by 2666 blocks, with a similar number of blocks for the oblique magnetic field. Because every block consists of $8 \times 8$ numerical cells, this number of blocks corresponds to 170624 numerical cells. This results in the finest (poorest) resolution of $\Delta x=\Delta y \approx 19.5 \mathrm{~km}(\Delta x=\Delta y \approx 625 \mathrm{~km})$ in the region below $y=3.7 \mathrm{Mm}$ (above $y=9 \mathrm{Mm}$ ) at $t=0$ seconds (see Figure 2). The refinement strategy is based on controlling numerical errors in mass density, which results in an excellent resolution of steep spatial profiles and greatly reduces numerical diffusion at these locations. The duration of a typical numerical run was 1500 seconds. Although the numerical simulations were carried out for $-5 \leq x \leq 5 \mathrm{Mm},-1 \leq y \leq 19 \mathrm{Mm}$, only the results in the regions of interest are displayed here.

\subsection{Vertical Equilibrium Magnetic Field: $\hat{\mathbf{s}}=\hat{\mathbf{y}}$}

First, we investigate the case of the vertical equilibrium magnetic field, which corresponds to $\hat{\mathbf{s}}=\hat{\mathbf{y}}$ in Equation (5). Here $\hat{\mathbf{y}}$ is a unit vector along the $y$-direction. The initial pulse of 
Figure 2 Numerical blocks with their boundaries (solid lines) and the pulse in velocity of Equation (12) (color maps) at $t=0$ seconds for the vertical and horizontal equilibrium magnetic fields. The maximum velocity (red color) corresponds to $0.2 \mathrm{~km} \mathrm{~s}^{-1}$.
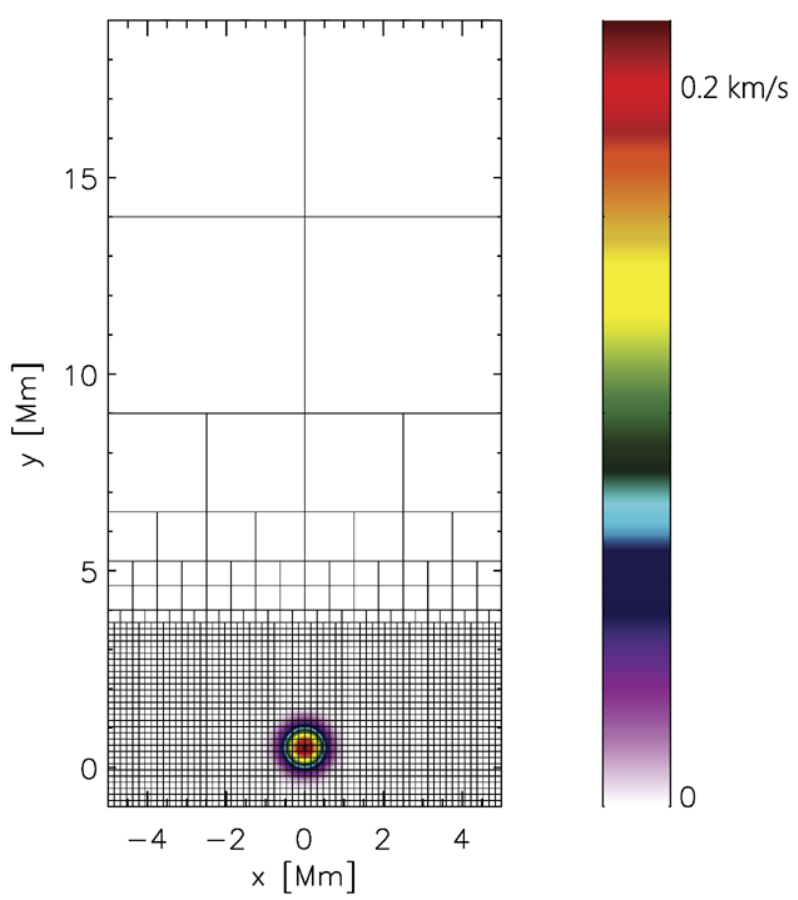

Equation (12) triggers magnetoacoustic-gravity waves in the solar atmosphere. These waves are compressive, and so we can trace their evolution in both temperature and velocity. Figure 3 illustrates logarithmic temperature profiles (color maps) and velocity vectors resulting from the perturbations. At $t=200$ seconds (top-left panel), the excited perturbations in the vertical component of velocity have already penetrated the solar corona, arriving at the point $(0,5.5) \mathrm{Mm}$. The perturbation in the vertical-velocity component propagates by $\approx 5 \mathrm{Mm}$ in the first 200 seconds of its evolution, which gives the speed of propagation as $\approx 25 \mathrm{~km} \mathrm{~s}^{-1}$. The average sound speed in this region is significantly higher. This is also confirmed by Fedun, Shelyag, and Erdélyi (2011) (cf. their Figures 4-7), who reported that their waves propagated from the transition region up to $2 \mathrm{Mm}$ within about 40 seconds of the simulation time. From this comparison we infer that the perturbation we initially imposed triggers flows in the solar atmosphere first, and thereafter these flows produce magnetoacousticgravity waves. At this moment in time, the transition region experiences a weak perturbation and the signal reflected from the transition region is represented by arrows around $x=0 \mathrm{Mm}, y=1.5 \mathrm{Mm}$. This reflected signal clashes with the slowly upwardly propagating wave that is triggered at the launching place, i.e., $x=0 \mathrm{Mm}, y=0.5 \mathrm{Mm}$. As a result of this clash, the leading front is generated. Cold chromospheric plasma lags behind, and at $t=400$ seconds (Figure 3, top-right panel) it reaches an altitude of $y \approx 6.5 \mathrm{Mm}$. The cold material is lifted because of the pressure gradient, which works against gravity and forces the chromospheric material to penetrate the solar corona (Sterling and Hollweg, 1989). At $t=800$ seconds (Figure 3, bottom-left), the transition region experiences well-developed oscillations whose central regions reach $y \approx 7 \mathrm{Mm}$ at $x=0 \mathrm{Mm}$, and also off-side propagating oscillations. These oscillations are of lower amplitudes at $t=1000$ seconds (Figure 3, bottom-right panel). At this time, the central plasma experiences gravitational deceleration while the region of cold plasma widens. The jet, which is associated with the shock, lasts for several hundred seconds. Its velocity reaches a maximum of $\approx 60 \mathrm{~km} \mathrm{~s}^{-1}$. 

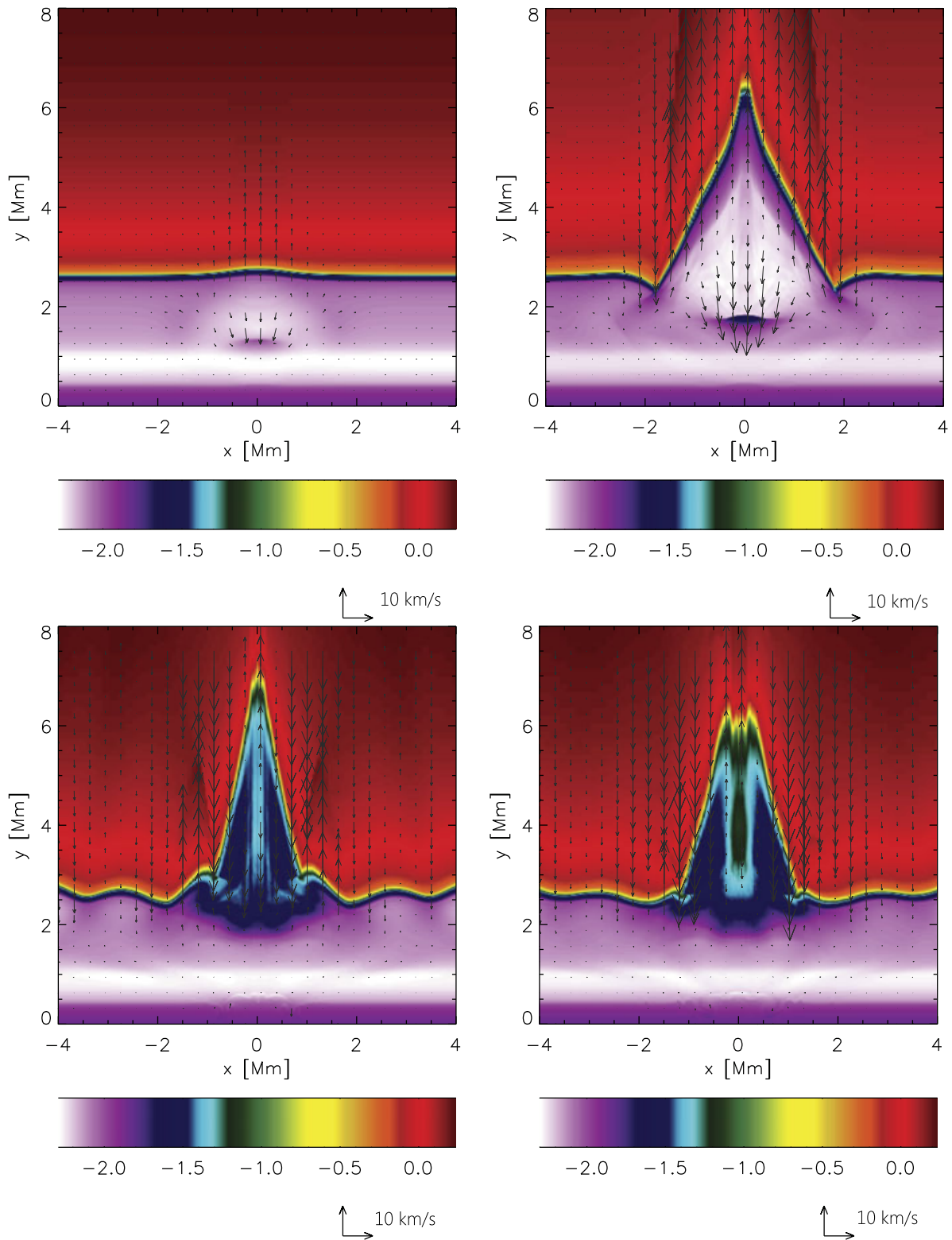

Figure 3 Spatial profiles of the logarithm of the temperature (color maps) and velocity (arrows) profiles at $t=200$ seconds (top-left), $t=400$ seconds (top-right), $t=800$ seconds (bottom-left), $t=1000$ seconds (bottom-right), for the vertical equilibrium magnetic field. Temperature is expressed in units of $1 \mathrm{MK}$. The arrow below each panel represents the length of the velocity vector, expressed in units of $10 \mathrm{~km} \mathrm{~s}^{-1}$. The corresponding movie can be found in fig3.avi in the Electronic Supplementary Materials.

Figure 4 illustrates the temporal variation of the vertical velocity component at the detection point $(0,5) \mathrm{Mm}$ for the case of Figure 3. As a result of the rapid mass density decrease with height, upwardly propagating waves grow in amplitude and steepen rapidly to form 
Figure 4 Temporal signature of $V_{y}$ at the point $(0,5) \mathrm{Mm}$ for the vertical equilibrium magnetic field.

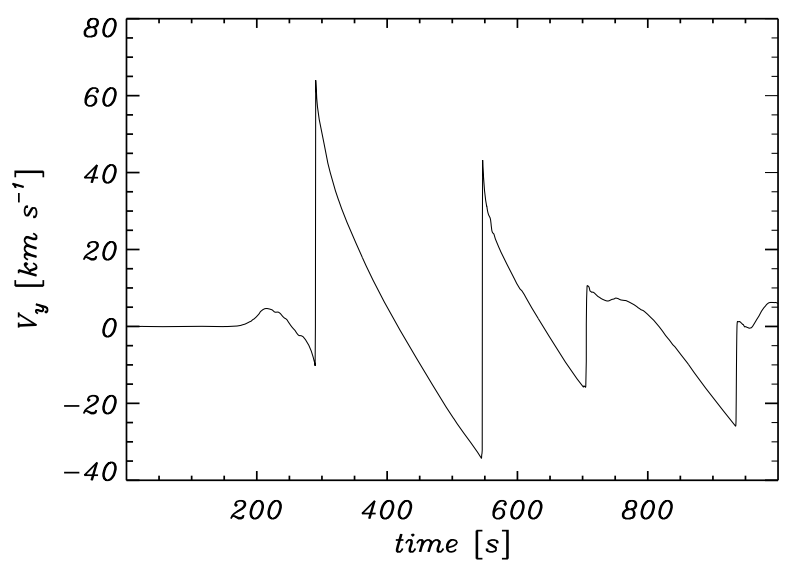

shocks. The arrival of the first shock-front at the detection point is clearly seen at $t \approx 290$ seconds. The second shock-front reaches the detection point at $t \approx 540$ seconds, i.e. approximately 250 seconds later. This secondary shock (and subsequent shocks that arrive after about 160 seconds and 240 seconds) results from the nonlinear wake, which lags behind the leading shock (Sterling and Hollweg, 1989; Srivastava and Murawski, 2011). These times can be compared with the acoustic cut-off period (Roberts, 2006),

$$
P_{\mathrm{ac}}(y)=\frac{4 \pi \Lambda(y)}{c_{\mathrm{s}}(y) \sqrt{1+2 \Lambda^{\prime}(y)}}
$$

which for $y=0.5 \mathrm{Mm}$ has a value of $P_{\mathrm{ac}}(y) \approx 180$ seconds. This value differs by $70 \mathrm{sec}-$ onds from the time-span between arrivals of neighboring shocks, which is 250 seconds. However, the 2D model we discuss here is more complex than the 1D scenario described by the Klein-Gordon equation (Roberts, 2006). The wave period we detected is altered by the interaction between up-going waves from the launching place of the initial pulse and the reflected wave from the transition-region signals. Because wave reflections originate at the large temperature gradients, the chromosphere sustains a cavity for these waves, which are represented by the oblique stripes located at altitudes of $0.7 \mathrm{Mm}<y<1.4 \mathrm{Mm}$ (Figure 5). In the neighborhood of the point $(0.4,0.4) \mathrm{Mm}$ we observe the formation of vortices (Figure 5, top panel) that experience an energy cascade into smaller scales. These vortices are present until the end of our simulation runs (Figure 5, bottom panel). The first vortex is triggered by the initial pulse in $V_{y}$, which is a characteristic feature of velocity perturbations. This first vortex causes convection in the convectively unstable plasma layers. According to Schwarzschild's instability condition, a medium is convectively unstable if the squared buoyancy (or Brunt-Väisälä) frequency,

$$
\omega_{g}^{2} \equiv-g\left(\frac{1}{\gamma \Lambda}-\frac{1}{\Lambda}\right)=\frac{g}{\Lambda}-\frac{g^{2}}{c_{\mathrm{S}}^{2}},
$$

is negative (e.g. Roberts, 2006). Because this criterion is satisfied for $y<0.6 \mathrm{Mm}$, convection sets in there. Such vortices were also described by Konkol, Murawski, and Zaqarashvili (2011), in a similar context. 
Figure 5 Total velocity [|V|] (color map) and velocity (arrows) profiles at $t=400$ seconds (top) and $t=1000$ seconds (bottom) for the vertical equilibrium magnetic field. The velocity vectors are expressed in units of $1 \mathrm{~km} \mathrm{~s}^{-1}$. The corresponding movie can be found in fig5.avi in the Electronic Supplementary Materials.
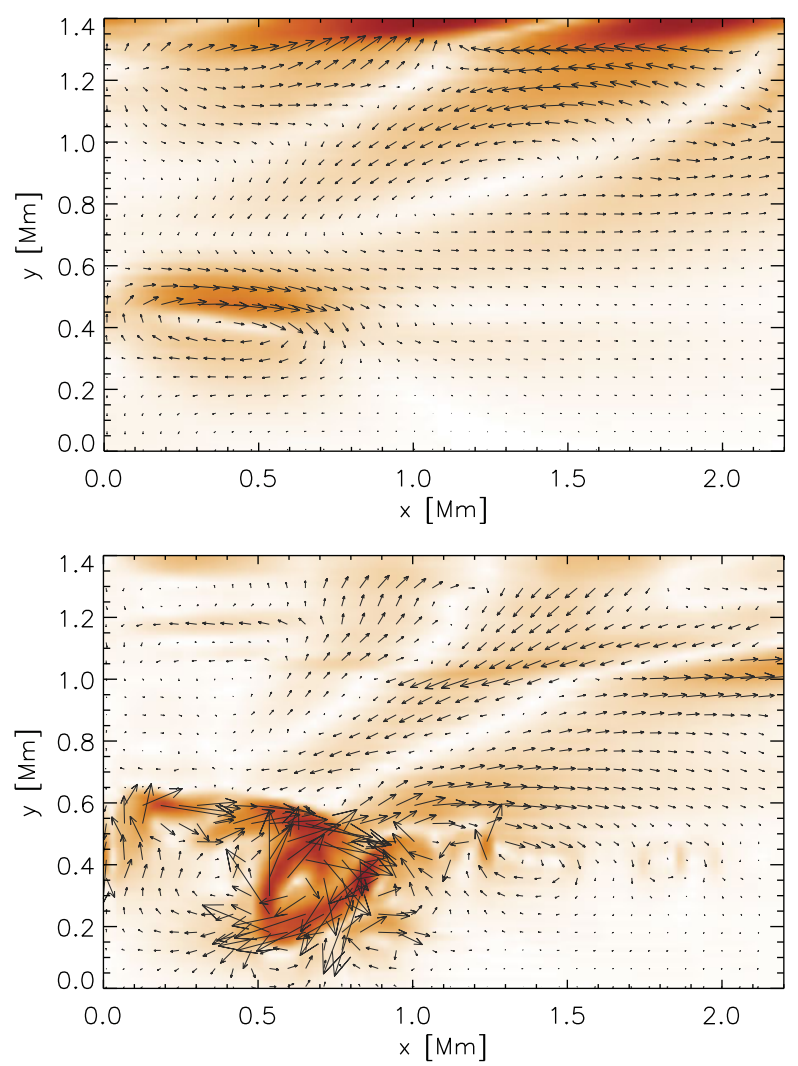

$1 \mathrm{~km} / \mathrm{s}$

\subsection{Horizontal Equilibrium Magnetic Field: $\hat{\mathbf{s}}=\hat{\mathbf{x}}$}

In this section, we investigate the horizontal equilibrium magnetic field, which corresponds to $\hat{\mathbf{s}}=\hat{\mathbf{x}}$ in Equation (5). In this case, the spatial profiles of $T$ drawn at $t=250$ seconds and $t=1000$ seconds (Figure 6) reveal small-amplitude oscillations of the transition region without the jet that was observed for the vertical magnetic field. Comparing this with Figure 3 , we see that the waves resulting from the initial pulses already arrived at the solar corona at $t=250$ seconds (Figure 6, left panel). The magnetic-field lines are horizontal, therefore these waves are essentially fast magnetoacoustic-gravity waves in the region of low plasma $\beta$ that occurs for $y>2.6 \mathrm{Mm}$ (Figure 1, bottom panel). It is interesting that the orientation of the magnetic field plays a very crucial role in determining the amplitude of transition-region oscillations.

Similar to the temporal-signatures of Figure $4, V_{y}$ at the detection point $(0,5) \mathrm{Mm}$ reveals the shocks. However, we now observe only two shocks for the horizontal magnetic field (as seen in Figure 7). The first shock arrives at the detection point at $t=250$ seconds and the second shock reaches this point at $t=400$ seconds. We note that the time-span of 150 seconds between these events is 100 seconds shorter than for the vertical magnetic field in Section 3.1. However, it is shorter by only 30 seconds than the acoustic cut-off period 

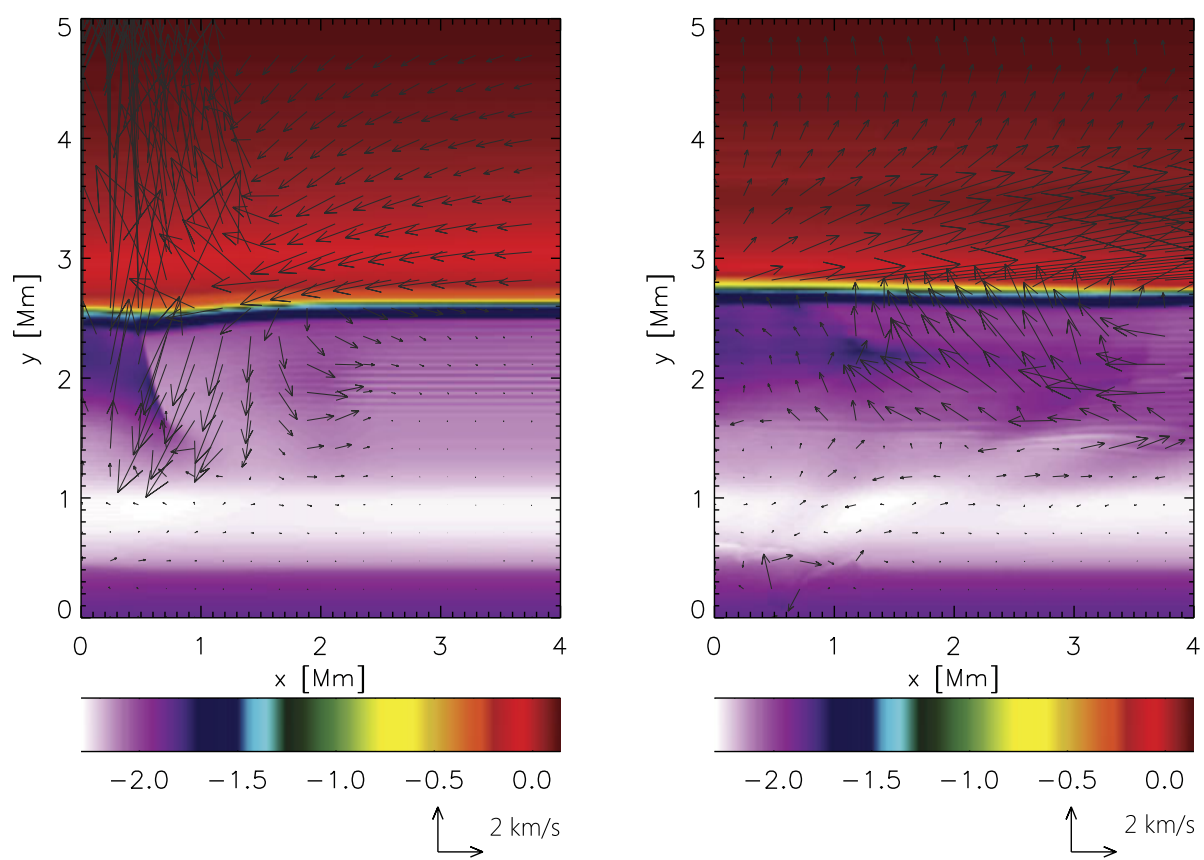

Figure 6 Spatial profiles of the logarithm of the temperature (color maps) and velocity (arrows) at $t=250$ seconds (top) and $t=1000$ seconds (bottom) for the horizontal equilibrium magnetic field. Temperature is in units of $1 \mathrm{MK}$. The arrow below each panel represents the length of the velocity vector, expressed in units of $2 \mathrm{~km} \mathrm{~s}^{-1}$. The corresponding movie can be found in fig6.avi in the Electronic Supplementary Materials.

Figure 7 Time signature of $V_{y}$ at the point $(0,5) \mathrm{Mm}$ for the horizontal equilibrium magnetic field.

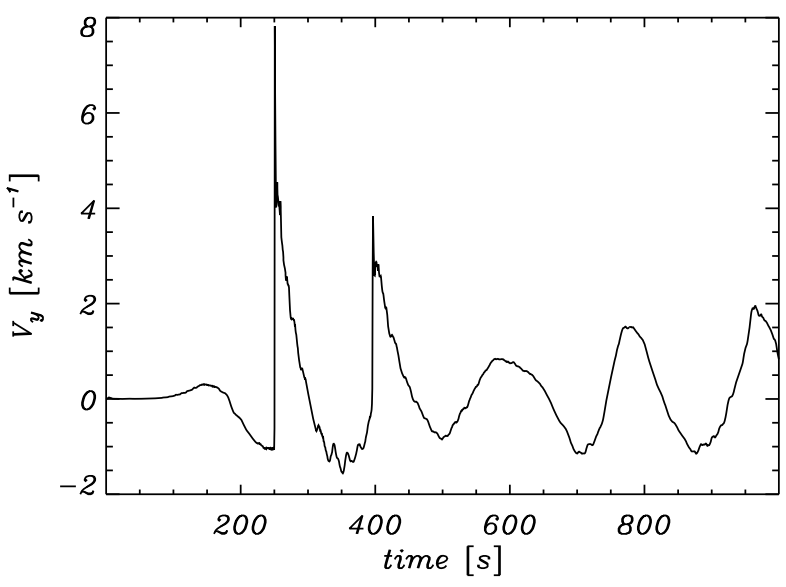

$\left[P_{\mathrm{ac}}\right]$ resulting from Equation (14). The subsequent three oscillations, which are of smaller amplitude, reveal periods on the order of $P_{\mathrm{ac}}$.

The chromosphere exhibits essentially similar features to those seen for the vertical magnetic field in Section 3.1. The vortex motion at $(x=0.4, y=0.4) \mathrm{Mm}$ and the trapped waves in the solar chromosphere for $0.7 \mathrm{Mm}<y<1.4 \mathrm{Mm}$ are again discernible (Figure 8). At $t=400$ seconds (top panel), the vortex is clearly developed. It is similar to that of Figure 5 
Figure 8 Total velocity [|V|] (color map) and velocity (arrows) profiles at $t=400$ seconds (top) and $t=1000$ seconds (bottom) for the horizontal equilibrium magnetic field. The velocity vectors are expressed in units of $1 \mathrm{~km} \mathrm{~s}^{-1}$. The corresponding movie can be found in fig8.avi in the Electronic Supplementary Materials.
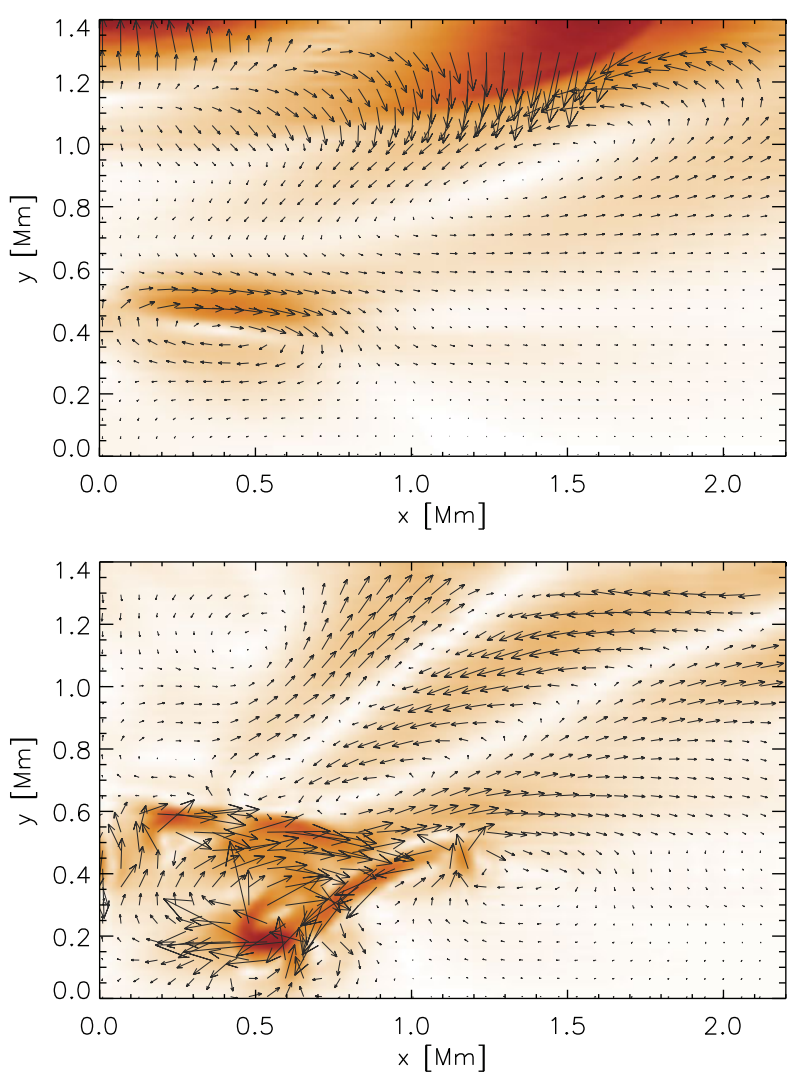

$1 \mathrm{~km} / \mathrm{s}$

(top panel). The flow patterns at the line $y=1.4 \mathrm{Mm}$ in Figures 5 and 8 differ in some details, though. In the case of the vertical magnetic field, the resulting waves are essentially slow magnetoacoustic-gravity waves, while Figure 8 corresponds to the horizontal background magnetic field with much contribution from fast magnetoacoustic-gravity waves. At a later time, turbulence results from this vortex, and it differs in some details from its analog of Figure 5 (bottom panel). In both the vertical and horizontal magnetic fields, the turbulence originates from the impulsively triggered perturbations that are initially launched in the convectively unstable atmospheric layers.

\subsection{Oblique Equilibrium Magnetic Field: $\hat{\mathbf{s}}=(\hat{\mathbf{x}}+\hat{\mathbf{y}}) / \sqrt{2}$}

In this section, we discuss the case of the oblique magnetic field, for which $\hat{\mathbf{s}}$ in Equation (5) is at an angle of $\pi / 4$ to the horizontal axis. Figure 9 displays temperature profiles at $t=400$ seconds (top-left panel), $t=600$ seconds (top-right panel), $t=800$ seconds (bottom-left panel), and $t=1000$ seconds (bottom-right panel). These profiles are more complex than those for either the horizontal or the vertical magnetic field of Sections 3.1 and 3.2. At $t=400$ seconds (Figure 9, top-left), we clearly see the rising transition region. At $t=1000$ seconds (Figure 9, bottom-right), we observe surface waves propagating along the transition region. However, for the oblique equilibrium magnetic field, we find that the temporal 

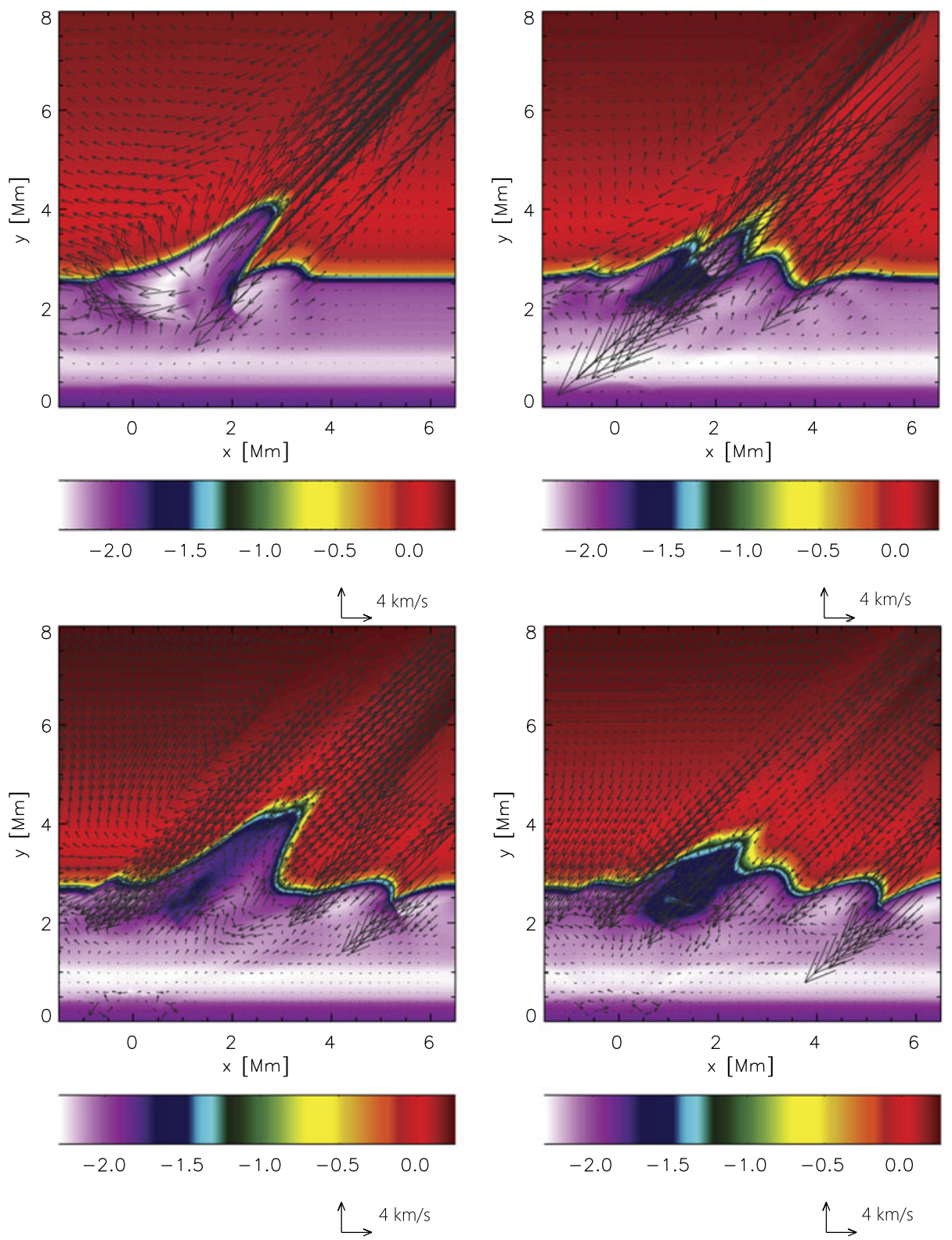

Figure 9 Spatial profiles of the logarithm of the temperature (color maps) and velocity (arrows) profile at $t=400$ seconds (top-left), $t=600$ seconds (top-right), $t=800$ seconds (bottom-left), $t=1000$ seconds (bottom-right) for the oblique equilibrium magnetic field. The temperature is given in units of $1 \mathrm{MK}$. The arrow below each panel represents the length of the velocity vector, expressed in units of $4 \mathrm{~km} \mathrm{~s}^{-1}$. The corresponding movie can be found in fig9.avi in the Electronic Supplementary Materials.

signatures of $V_{y}$ reveal shocks, and the periods are about 250-300 seconds (Figure 10). The jet, which is associated with the shock, lasts for several hundred seconds and reaches a velocity maximum of $\approx 30 \mathrm{~km} \mathrm{~s}^{-1}$. This is a result of complex interaction of the waves 
Figure 10 Time signature of $V_{y}$ at the points $(4.5,5) \mathrm{Mm}$ (top) and $(3.84,4.98) \mathrm{Mm}$ (bottom) for the oblique equilibrium magnetic field.
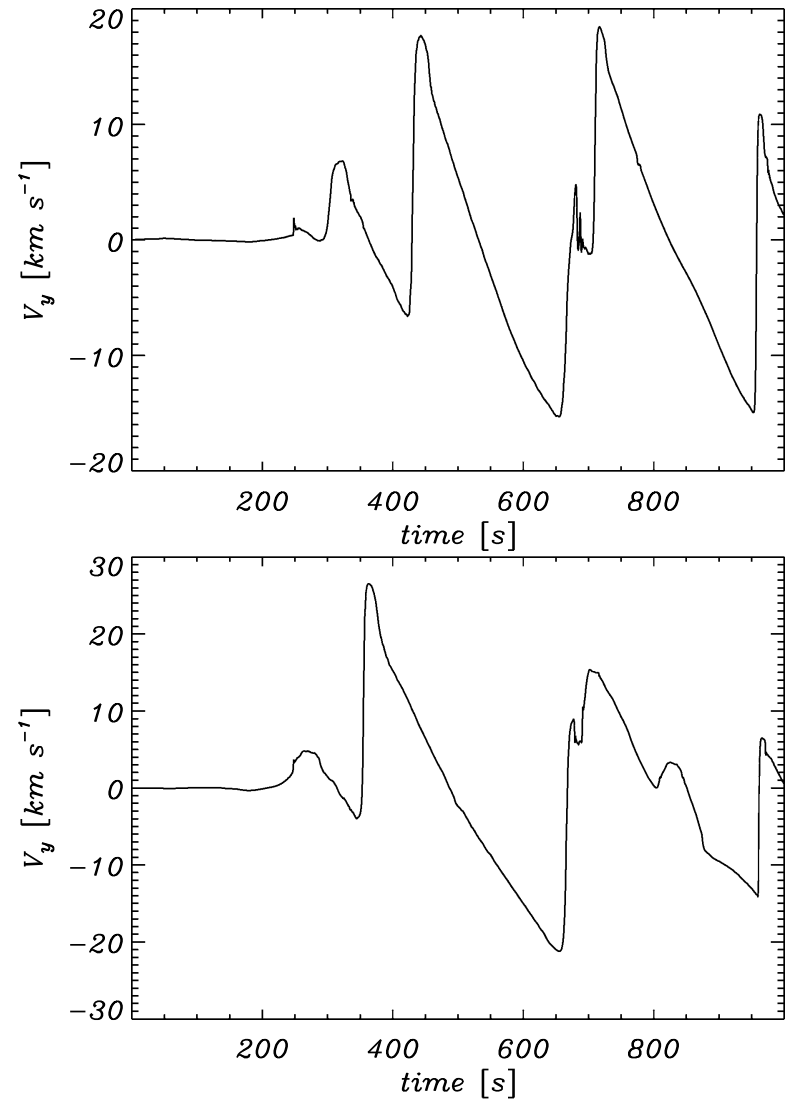

Figure 11 Total velocity [|V|] (color map) and velocity (arrows) profile at $t=1000$ seconds for the oblique equilibrium magnetic field. The velocity vectors are expressed in units of $1 \mathrm{~km} \mathrm{~s}^{-1}$. The corresponding movie can be found in fig11.avi in the Electronic Supplementary Materials.
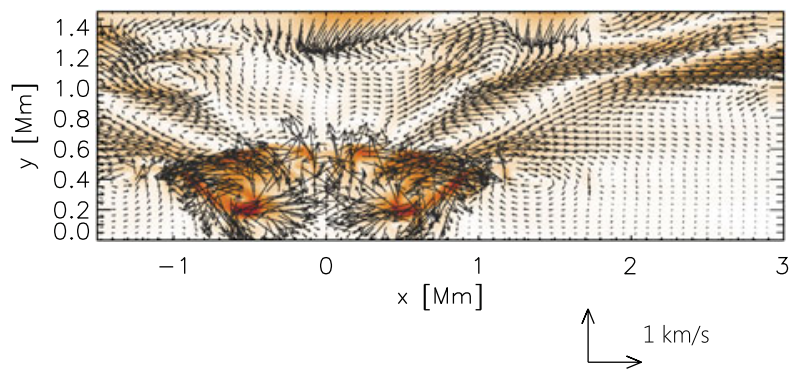

with the background plasma in the upper regions of the solar atmosphere. This complexity is seen at $t=1000$ seconds in the velocity profiles of Figure 11, which clearly illustrates the trapped and reflected waves in the solar chromosphere as well as clearly developed vortices.

\section{Discussion and Conclusion}

We have investigated the impulsive excitation of magnetoacoustic-gravity oscillations and compared and contrasted the resulting propagation under different orientations of equi- 
librium magnetic fields. We performed 2D numerical simulation of the velocity and gaspressure pulses, which mimic a solar granule. These pulses were initially launched at the top of the solar photosphere in a stratified solar atmosphere using the VAL-C temperature profile. We find that in the cases where the background magnetic field possesses a non-zero vertical component, the amplitude of the upwardly propagating perturbations rapidly grows with height due to the rapid decrease in the equilibrium mass density. Therefore, the perturbation quickly steepens into shocks in the upper regions of the solar chromosphere, which launches the cool material behind it.

We found that the excitation of magnetoacoustic-gravity waves in a non-horizontal equilibrium magnetic-field configuration is channeled upwards and able to cause large-amplitude transition-region oscillations. Meanwhile, the horizontal equilibrium magnetic field configuration results in relatively smaller-amplitude transition-region oscillations caused by fast magnetoacoustic-gravity waves. These spread their energy across magnetic-field lines, in contrast to slow magnetoacoustic-gravity waves, which in a strongly magnetized plasma region are guided along the magnetic-field lines. This indicates that the wave energy is transferred into the upper atmosphere in larger amounts when the magnetic field is more vertical. However, the complexity and therefore the evolution of a horizontal equilibrium magnetic field allows the reflection and trapping of the waves in the lower solar atmosphere and can influence the localized dynamics and heating of the atmosphere. The reflection of the waves in the lower solar atmosphere and its trapping may also cause chromospheric cavities.

By analyzing the temporal-signature of $V_{y}$ at a fixed spatial point, we also found that the oscillation period is longer when comparing the vertical magnetic field (here, the characteristic oscillation period was $\approx 250-300$ seconds) with the horizontal equilibrium magnetic system (in which the characteristic period of oscillation was $\approx 150-200$ seconds). For the vertical magnetic-field system in low plasma- $\beta$ regions, the magnetoacoustic-gravity waves are adequately described as slow magnetoacoustic-gravity waves (propagating along the magnetic-field lines at approximately the sound speed $\left[c_{\mathrm{s}}(y)\right]$, see Equation (7)). For the horizontal magnetic-field system in strongly magnetized plasma, the oscillations transverse to the magnetic field are appropriately described as fast magnetoacoustic-gravity waves (propagating across the magnetic-field lines at the fast speed, $c_{\mathrm{f}}^{2}(y)=c_{\mathrm{A}}^{2}(y)+c_{\mathrm{s}}^{2}(y)$, see Equations (6) and (7)). For $y=0.5 \mathrm{Mm}$ we have $c_{\mathrm{A}} \ll c_{\mathrm{s}}$; the cut-off frequencies of the fast and slow magnetoacoustic-gravity waves are very close to each other, which results in very close values of the cut-off periods. Therefore, we might expect that the periods detected for the vertical and horizontal magnetic fields exhibit similar values. However, the cut-off periods are derived on the basis of the linear theory, which is valid for small-amplitude oscillations only. Such small oscillations are present in the case of the horizontal magnetic field, therefore the numerical data lie close to the analytical prediction. In the vertical magnetic field, the upwardly propagating waves interact with the waves that are reflected from the transition region. This gives rise to larger-amplitude oscillations, which significantly alter the background plasma. The waves that propagate through this strongly modified medium exhibit modified velocities, and they are reflected from the transition region, which is locally strongly curved. This results in periods within the range 250-300 seconds, which differ from $P_{\text {ac }}$.

It is known that radiation is an effective mechanism of wave damping in the low photosphere (Mihalas and Toomre, 1982). It might not radically alter the system's behavior, but radiative damping is at least likely to reduce the amplitude of the waves that reach the transition region, which leads to shorter jets than those that we see in our models, and also to lower amplitudes of the coronal shocks. We did not invoke radiative cooling or thermal conduction in our model atmosphere, because we aimed to model the small-scale atmospheric 
regions above the solar photosphere where these effects are not believed to be dominant. However, we intend to include these effects in our future studies.

The solar atmosphere is structured by convective overshoot, which is absent from our model. Instead, we isolated a single granule-like perturbation with the aim to mimic a magnitude of flow and plasma temperature that is associated with the solar granulation (Baran, 2011). We considered the complex scenario that results from this simple model to understand the physics of wave phenomena above such magnetic structures in the solar atmosphere. We intend to develop more advanced models in our future studies.

In conclusion, our numerical simulations clearly demonstrate that small-amplitude initial pulses in vertical velocity and gas pressure are able to trigger a plethora of dynamic phenomena in the upper regions of the solar atmosphere with periods in the range 150-300 seconds, a value that depends on the orientation of the background magnetic field. However, it should be noted that our 2D simulations are idealized in the sense that they do not include radiative transfer and thermal conduction along field lines. The magnetic-field configuration and the equilibrium stratification are simple and we modeled a single granule only. These limitations require additional studies, which we intend to carry out in the near future.

Acknowledgements The authors express their thanks to the referee for the stimulating comments. This work has been supported by a Marie Curie International Research Staff Exchange Scheme Fellowship within the 7th European Community Framework Program (KM). The software used in this work was in part developed by the DOE-supported ASC/Alliance Center for Astrophysical Thermonuclear Flashes at the University of Chicago. JM acknowledges IDL support provided by STFC, UK. RO acknowledges financial support from MICINN/MINECO and FEDER funds through grant AYA2011-22846 and also CAIB through the "Grups Competitius" scheme and FEDER funds. KM expresses his thanks to Kamil Murawski for his assistance in drawing the numerical data.

Open Access This article is distributed under the terms of the Creative Commons Attribution License which permits any use, distribution, and reproduction in any medium, provided the original author(s) and the source are credited.

\section{References}

Baran, O.A.: 2011, In: Choliy, V., Ivashchenko, G., Ivaniuk, O. (eds.) 2010, Adv. Astron. Space Phys., Proc. the 17th Young Scientists' Conference on Astron. Space Phys. 53.

Bogdan, T.J., Carlsson, M., Hansteen, V.H., McMurry, A., Rosenthal, C.S., Johnson, M., Petty-Powell, S., Zita, E.J., Stein, R.F., McIntosh, S.W., Nordlund, A.: 2003, Astrophys. J. 599, 626.

Centeno, R., Socas-Navarro, H., Lites, B., Kubo, M., Frank, Z., Shine, R., Tarbell, T., Title, A., Ichimoto, K., Tsuneta, S., Katsukawa, Y., Suematsu, Y., Shimizu, T., Nagata, S.: 2007, Astrophys. J. Lett. 666, L137.

Fedun, V., Erdélyi, R., Shelyag, S.: 2009, Solar Phys. 258, 219. doi:10.1007/s11207-009-9407-9.

Fedun, V., Shelyag, S., Erdélyi, R.: 2011, Astrophys. J. 727, 17.

Fedun, V., Verth, G., Jess, D.B., Erdélyi, R.: 2011, Astrophys. J. Lett. 740, L46.

Gruszecki, M., Murawski, K., Kosovichev, A.G., Parchevsky, K.V., Zaqarashvili, T.: 2011, Acta Phys. Pol. 42, 1333.

Hasan, S.S., van Ballegooijen, A.A., Kalkofen, W., Steiner, O.: 2005, Astrophys. J. 631, 1270.

Katsukawa, Y., Berger, T.E., Ichimoto, K., Lites, B.W., Nagata, S., Shimizu, T., Shine, R.A., Suematsu, Y., Tarbell, T.D., Title, A.M., Tsuneta, S.: 2007, Science 318, 1594.

Konkol, P., Murawski, K., Zaqarashvili, T.V.: 2011, Astron. Astrophys. 537, A96.

Kostik, R., Khomenko, E., Shchukina, N.: 2009, Astron. Astrophys. 506, 1405.

Krijger, J.M., Rutten, R.J., Lites, B.W., Straus, Th., Shine, R.A., Tarbell, T.D.: 2001, Astron. Astrophys. 379, 1052.

Kuridze, D., Zaqarashvili, T.V., Shergelashvili, B.M., Poedts, S.: 2008, Ann. Geophys. 26, 2983.

Kuridze, D., Zaqarashvili, T.V., Shergelashvili, B.M., Poedts, S.: 2009, Astron. Astrophys. 505, 763.

Lee, D., Deane, A.E.: 2009, J. Comput. Phys. 228, 952.

McAteer, R.T.J., Gallagher, P.T., Williams, D.R., Mathioudakis, M., Phillips, K.J.H., Keenan, F.P.: 2002, Astrophys. J. Lett. 567, L165. 
McAteer, R.T.J., Gallagher, P.T., Williams, D.R., Mathioudakis, M., Bloomfield, D.S., Phillips, K.J.H., Keenan, F.P.: 2003, Astrophys. J. 587, 806.

McIntosh, S.W., Judge, P.G.: 2001, Astrophys. J. 561, 420.

Mihalas, B.W., Toomre, J.: 1982, Astrophys. J. 263, 386.

Murawski, K., Srivastava, A.K., Zaqarashvili, T.V.: 2011, Astron. Astrophys. 535, A58.

Rieutord, M., Ludwig, H.G., Roudier, T., Nordlund, A., Stein, R.: 2002, Nuovo Cimento C 025, 523.

Roberts, B.: 2006, Phil. Trans. Roy. Soc. London A 364, 447.

Schrijver, C.J., Title, A.M.: 2003, Astrophys. J. Lett. 597, L165.

Shibata, K., Magara, T.: 2011, Living Rev. Solar Phys. 8, 6. http://solarphysics.livingreviews.org/open?pubNo= Irsp-2011-6\&amp;page=articlese8.html.

Shibata, K., Nakamura, T., Matsumoto, T., Otsuji, K., Okamoto, T.J., Nishizuka, N., et al.: 2007, Science 318, 1591.

Srivastava, A.K.: 2010, New Astron. 15, 621.

Srivastava, A.K., Murawski, K.: 2011, Astron. Astrophys. 534, A62.

Srivastava, A.K., Kuridze, D., Zaqarashvili, T.V., Dwivedi, B.N.: 2008, Astron. Astrophys. Lett. 481, L95.

Srivastava, A.K., Zaqarashvili, T.V., Kumar, P., Khodachenko, M.L.: 2010, Astrophys. J. 715, 292.

Sterling, A.C., Hollweg, J.V.: 1989, Astrophys. J. 343, 985.

Vecchio, A., Cauzzi, G., Reardon, K.P.: 2009, Astron. Astrophys. 494, 269.

Vecchio, A., Cauzzi, G., Reardon, K.P., Janssen, K., Rimmele, T.: 2007, Astron. Astrophys. Lett. 461 , L1.

Vernazza, J.E., Avrett, E.H., Loeser, R.: 1981, Astrophys. J. 45, 635.

Zhang, J., Cheng, X., Ding, M.D.: 2012, Nat. Commun. 3, 747. 